\title{
Hemostasis profile in COVID-19 infection
}

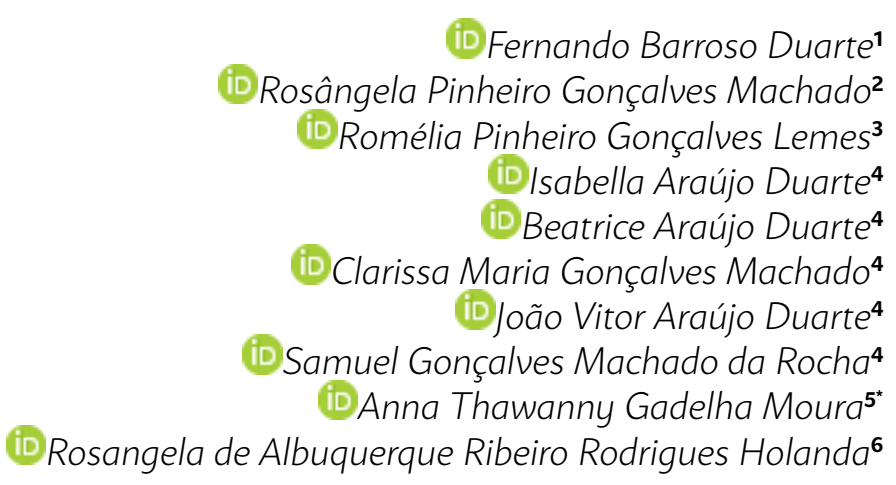

1. Departamento de Cirurgia - Universidade federal do Ceará, Fortaleza, CE, Brasil. 2. Centro de Ciências da Saúde, Departamento de Medicina - Universidade de Fortaleza (UNIFOR), Fortaleza, CE, Brasil. 3. Departamento de Análises Clínicas e Toxicológicas (DACT) - Universidade federal do ceará, Fortaleza, CE, Brasil. 4. Faculdadse de Medicina -Faculdade Christus, Fortaleza, CE, Brasil. 5. Laboratório de Pesquisa em Hemoglobinopatias e Doenças Genéticas e Hematológicas - Universidade Federal do Ceará, Fortaleza, CE, Brasil. 6. Hospital Universitário Walter Cantídio - Universidade Federal do Ceará, Fortaleza, CE, Brasil.
Dear Editor,

According to some current studies, the infection by COVID-19 (SARS- CoV-2) appears to be associated with the involvement of hemostasis in the Chinese population, developing to Acute Respiratory Distress Syndrome (ARDS). The context reported here focuses on cases with ARDS, which has been implicated with the presence of an endothelial lesion, production of pro-coagulating factors, and generation of fibrin degradation product (D-dimer), compatible with disseminated intravascular coagulation (DIC). ${ }^{1}$ The increase in the concentration of D-dimer 3-4 times above the normal levels was associated with the outcome of death and there are reports of thromboembolic phenomena, and factors of worse prognosis, such as advanced age, low immunity, neutrophilia, and organ and coagulation dysfunction. ${ }^{2}$ Patients who develop a more severe response to the disease may evolve to dyspnea and hypoxemia within one week from the onset of the disease and may progress rapidly to ARDS or target organ failure. Among other possibilities, coagulation, in the cases that have been reported in the literature, consists of prolonged prothrombin time (PT) and partial activated thromboplastin time (APTT) in $2.1 \%$ and $9.7 \%$, respectively, of the patients who had ARDS. Epidemiological data report that changes in coagulation, in COVID-19 infections, associated with ARDS and the systemic inflammatory process, may react releasing a large amount of pro-inflammatory 
cytokines - tumor necrosis factor, interleukin (IL) -1, IL -6 and IL-8 - although the pathogenesis of human COVID19 has not yet been fully clarified. Studies reinforce the hypothesis that the great increase in the production of cytokines is associated with a deficient immune system and are the two main triggers of events that culminate in the serious evolution of the infection..$^{1,2}$ In this context, it is important to develop preventive measures, as well as laboratory tests and early prognostic markers of patients who will present the severe form, so that a therapeutic approach is performed in the initial phase in patients affected by COVID-19. This way, it will be possible to avoid the evolution to death in this group of patients, since the course of the disease can be quick, progressing to ARDS within one week from the onset of the disease, in addition to target organ failure. ${ }^{2}$ Comparing the laboratory tests of patients with and without ARDS, it was noticed that the group with ARDS had significant changes in index values, i.e., liver injury, renal dysfunction, related to inflammation and coagulation function. Also, the lymphocyte count and CD8 T cells were significantly decreased. In addition to this worrying matter, patients who developed ARDS did not respond well to treatments with antiretrovirals. ${ }^{2}$ Given these facts, it is of utmost importance to evaluate the benefit laboratory tests and prognostic markers, on hospital admission, could bring to favor those patients who will evolve to the severe form, aiming at early preventive and therapeutic measures, to avoid evolution to unfavorable outcomes. Among the important tests so far, we can mention blood count, PT, APTT, and D-dimer, which have all been mentioned and whose correlations were observed in the literature as prognostic markers, as follows: severe lymphopenia, neutrophilia, and thrombocytopenia at the complete blood count; extended PT and increased D-dimer are associated with cases of severe evolution. ${ }^{3-5}$ However, the guidance to patients who were already using oral anticoagulant medications, before contracting COVID19 , is to continue with the treatment; however, greater laboratory and clinical monitoring of these patients is necessary, as recommended by Chinese studies that note that altered PT, even at mild levels, is also a factor of poor prognosis and reinforce that, in this scenario, the patient/control relationship should be used instead of the INR.

\section{Acknowledgments}

We thank all health professionals at the Walter Cantídio University Hospital.

\section{Conflict of interest}

The authors have no competing interests.

PALAVRAS-CHAVE: COVID-19. Infecções por coronavirus. Hemostasia.

\section{REFERENCES}

1. Fan BE, Chong VCL, Chan SSW, Lim GH, Lim KGE, Tan GB, et al. Hematologic parameters in patients with COVID-19 infection. Am J Hematol. 2020;10.1002/ajh.25774. doi:10.1002/ajh.25774.

2. Wu C, Chen $X$, Cai $Y, X i a ~ J, Z h o u ~ X, X u ~ S$, et al. Risk factors associated with acute respiratory distress syndrome and death in patients with coronavirus disease 2019 pneumonia in Wuhan, China. JAMA Intern Med. 2020;e200994. doi:10.1001/jamainternmed.2020.0994.

3. Zhou F, Yu T, Du R, Fan G, Liu Y, Liu Z, et al. Clinical course and risk factors for mortality of adult inpatients with COVID-19 in Wuhan, China: a retrospective cohort study. Lancet. 2020;395(10229):1054-62.

4. Tang N, Li D, Wang X, Sun Z. Abnormal coagulation parameters are associated with poor prognosis in patients with novel coronavirus pneumonia. J Thromb Haemost. 2020;18(4):844-7.

5. Thachil I, Tang N, Gando S, Falanga A, Cattaneo M, Levi M, et al. ISTH interim guidance on recognition and management of coagulopathy in COVID-19. J Thromb Haemost. 2020;18(5):1023-6. 